\title{
Title: Emergence of drug resistance in the Swiss HIV Cohort Study under potent antiretroviral therapy is observed in socially disadvantaged patients.
}

\author{
Authors: Irene A. Abela1,2*, Alexandra U. Scherrer ${ }^{1,2 *}$, Jürg Böni², Sabine Yerly³, Thomas Klimkait5, \\ Matthieu Perreau ${ }^{6}$, Hans H. Hirsch ${ }^{8}$, Hansjakob Furrer ${ }^{9}$, Alexandra Calmy ${ }^{4}$, Patrick Schmid ${ }^{10}$, Matthias \\ Cavassini $^{7}$, Enos Bernasconi ${ }^{10,11}$, Huldrych F. Günthard ${ }^{1,2}$ and the Swiss HIV Cohort Study
}

\begin{abstract}
${ }^{1}$ Division of Infectious Diseases and Hospital Epidemiology, University Hospital Zürich, ${ }^{2}$ Institute of Medical Virology, University of Zurich, ${ }^{3}$ Laboratory of Virology, Division of Infectious Diseases, and ${ }^{4} \mathrm{HIV} /$ AIDS Unit, Infectious Disease Service, Geneva University Hospitals, ${ }^{5}$ Department of Biomedicine, University of Basel, Divisions of ${ }^{6}$ Immunology and Allergy, and ${ }^{7}$ Infectious Diseases, University Hospital Lausanne, ${ }^{8}$ Division of Infectious Diseases and Hospital Epidemiology, University Hospital Basel, ${ }^{9}$ Department of Infectious Diseases, Bern University Hospital, University of Bern, ${ }^{10}$ Division of Infectious Diseases, Cantonal Hospital St. Gallen,

${ }^{11}$ Division of Infectious Diseases, Regional Hospital Lugano, Switzerland
\end{abstract}

*these authors contributed equally

corresponding author: Irene A. Abela, Division of Infectious Diseases and Hospital Epidemiology, University Hospital Zürich and Institute of Medical Virology, University of Zurich (abela.irene@virology.uzh.ch)

Summary: In a case-control study we were able to identify specific risk factors for acquired drug resistance in all patients starting their first cART in the Swiss HIV Cohort Study (SHCS) since 1996.

This work presented in part at the Conference on Retroviruses and opportunistic infections 2018, CROI 2018 (Boston, March 4-7, 2018,) Poster ID 2102

(C) The Author(s) 2019. Published by Oxford University Press for the Infectious Diseases Society of America. All rights reserved. For permissions, e-mail: journals.permissions@oup.com. 


\section{Abstract}

Background The rate of acquired HIV-1 drug resistance (ADR) has fallen dramatically since introduction of combined antiretroviral therapy (cART) in Switzerland. However, clinical experience indicates that there are still patients at risk of newly acquiring drug resistance despite having access to cART. Here, we characterized risk factors for ADR, in order to improve patient care, prevent emergence of drug resistance, and treatment failure.

Methods We performed a case-control study to identify risk factors for ADR in all patients starting their first cART in the Swiss HIV Cohort Study (SHCS) since 1996. The SHCS is highly representative and includes over $75 \%$ of patients receiving ART in Switzerland. To this end, we implemented a systematic medical chart review to obtain more detailed information on additional parameters, which are not routinely collected in the SHCS. The collected data was analyzed univariable and multivariable conditional logistic regressions.

Results We included in our study 115 cases and 115 matched controls. Unemployment (mOR 2.9 (95\% CI: 1.3-6.4), p=0.008), African origin (mOR3.0 (95\%CI: 1.0-9.2), p=0.047), co-medication with anti-infectives (mOR 3.6 (95\%CI: 1.0-12.6), p=0.045) and symptoms of mental illness (mOR 2.6 (95\% CI: 1.2-5.5), $\mathrm{p}=0.012$ ) were associated with ADR in the multivariable model.

Conclusions Although, ADR has become very rare with cART due to new potent therapies, patients in socially challenging life situations or presenting with mental health issues are at higher risk for drug resistance. Prompt identification and adequate support of these patients before ADR will prevent treatment failure and HIV-1 transmission.

Keywords (5) HIV-1 drug resistance, risk factors, antiretroviral therapy, socioeconomic factors 


\section{Introduction}

Emergence of HIV- 1 drug resistance has become less frequent with the introduction of combination antiretroviral treatment (cART) [1-7] due to its high effectiveness at suppressing human immunodeficiency virus (HIV) replication in infected individuals [8-10].

Consequently, in resource-rich settings with continuous access to cART and HIV-related care, HIV infection has become a controllable chronic illness with life expectancy approaching that of the general population [11-13]. However, clinical experience indicates that there are still patient groups at risk of newly acquiring drug resistance despite having access to cART [14]. Several studies indicate that low socioeconomic status (SES) not only increases vulnerability to HIV infection and is associated with having poorer virological and immunological outcomes, but also impedes engagement and retention of HIVinfected persons in clinical care [15-20]. Some European studies have also shown that lower socioeconomic status is associated with non- adherence to ART [20]. Non-adherence and subsequent drug resistance therefore still poses a major obstacle to successful treatment and elimination of HIV- 1 transmission in resource-rich settings [7, 21, 22].

In contrast, in resource limited settings rising rates of acquired drug resistance may not only reflect poor adherence but also shortage of optimal regimens, and a lack of viral load monitoring. Cost and laboratory availability limit monitoring severely and pose the major hurdles for successful treatment [23]. For public health and prevention strategies, and to assess requirements for new drugs, it is important to monitor the spread of drug resistance in the HIV-infected population [7]. Moreover, in resource- rich settings, it is crucial to analyze risk factors leading to resistance in the presence of already highly active antiretroviral therapy. Once risk factors are identified, patients at risk can be followed up closely and support can be intensified.

In the current study, we retrospectively characterize risk factors and examine sociodemographic correlates for the development of drug resistance in a nation-wide sample of HIV-positive patients receiving cART after 1996. 


\section{Methods}

\section{Study population and design}

We performed a case-control study to identify risk factors for acquired HIV-1 drug resistance (ADR) in patients starting their first cART regimen in the Swiss HIV Cohort Study (SHCS). The SHCS is an ongoing, nationwide, multicenter, clinic-based, observational study with continuous enrollment and semiannual study visits. At least 75\% of all HIV-1 diagnosed adults receiving cART in Switzerland are enrolled in the SHCS. The SHCS has been approved by the ethical committees of all participating institutions, and written informed consent has been obtained from all participants [24]. The SHCS has a linked drug resistance database, which includes sequences from genotypic resistance tests (GRTs) performed by the four authorized laboratories in Switzerland. Over 12'000 sequences were generated retrospectively from the biobank to also cover treatment failing episodes and transmitted drug resistance, when drug resistance testing was not performed routinely [25]. Sequences are stored in a central database (SmartGene; Integrated Database Network System version 3.8.1). All laboratories performed population-based sequencing $[2,25]$. Acquired drug resistance has been defined as the occurrence of at least one major mutation listed by the International Antiviral Society-USA [22].

We restricted the analysis to patients who received potent cART defined as treatment with at least three drugs from two different drug classes [Nucleoside analog reverse-transcriptase inhibitors

(NRTI), Non-nucleoside reverse-transcriptase inhibitors (NNRTI), boosted Protease inhibitor (PI), integrase inhibitor, or entry inhibitor]. Patients who had in their medical history an exposure to mono/dual/triple NRTI therapies or regimens including unboosted PIs were excluded from the analysis.

We identified 115 cases that acquired a drug resistance mutation, proven by a GRT, after cART initiation. Subsequently, we randomly matched 115 controls (1:1) based on the following criteria: Plasma HIV-1 RNA at treatment initiation [ $<1000$ copies/mL, 1000-9999 copies/mL, $\geq 10000$ copies/mL], CD4 cell count at baseline (0-99 cells/ $\mu \mathrm{L}, 100-199$ cells/ $\mu \mathrm{L},>200$ cells $/ \mu \mathrm{L})$, year of cART initiation $[<1998,1999-2006$, >2006], presence of a baseline drug resistance mutation, and the SHCS study center (Basel, Berne, Geneva, Lausanne, Lugano, St. Gallen, Zürich) [Supplementary Table 1]. The latter was implemented to exclude 
documentation and treatment bias between the SHCS centers. The follow-up time of the controls (cART initiation until last follow-up) had to be at least as long as for the cases (cART initiation until detection of ADR).

\section{Potential risk factors for acquired drug resistance}

In addition to the routinely collected data in the SHCS [24] we hypothesized that additional factors which are not routinely documented in the six monthly questionnaire could be associated with acquired drug resistance mutations. Therefore, we additionally defined potential relevant factors relevant for acquiring drug resistance that were not documented in the SHCS protocol and subsequently performed a medical chart review on all 230 patients and collected detailed information beyond the documented SHCS questionnaire [Supplementary Table 2]. We not only looked at the medical chart documentation at the time point of GRT and the previous visits (all visits in the 6 months prior to GRT) but also screened all available admission letters and hospitalization documentation during the 6 months prior to GRT. We did not include any time points after GRT in our analysis. As depression is frequent among HIV infected persons $[26,27]$ and the presence of mental health disorders has been found to correlate with reduced adherence [28], we analyzed medical chart documentation for symptoms of mental illness, diagnosis of mental health disorders and for documented psychiatric treatment (psychiatric hospitalization, outpatient mental health treatment, treatment by a psychiatrist or psychiatric medication).

We systematically looked at the three monthly visit documentation up to 6 months prior to GRT.

To evaluate whether co-infections or complicated medication regimens (due to additional treatment of infections) may have led to adherence problems we screened for co-medication with anti-infectives in the 6 months prior to and at the time point of GRT. We screened whether the patients had an additional treatment with antibiotic, antimycobacterial, antifungal, antiviral (other than ART) or antiparasitic medication. Additionally we screened specifically what medication was prescribed and whether drugdrug interactions were expected. We also screened for documented ART drug level measurements (see Supplementary Table 2). 
Additionally, as we hypothesized that difficulties in communication and reduced information might largely affect adherence, we specifically looked for language barrier. Language barrier was defined when in the medical chart it was clearly stated that the patient and doctor could not communicate as they did not speak the same language. In the SHCS this applies to patients who do not speak French, Italian, German or English.

Self-reported adherence in the SHCS questionnaire has been previously validated to predict viral failure, HIV drug resistance and mortality [29-31].

\section{Statistical analysis}

We performed univariable and multivariable conditional logistic regression to identify risk factors for ADR. We included factors from the univariable model with a p-value $<0.1$ in the multivariable models or factors, which were previously known to be associated with drug resistance. We systematically looked for collinear factors. If identified, we have only included one of these factors in the multivariable model. We performed sensitivity analyses including only patients with a perfect match based on the abovementioned criteria. We did not include self-reported adherence in the final multivariable analysis, only in a sensitivity analysis, as adherence is most likely on the causal path towards acquired drug resistance. We performed statistical analyses with Stata (version 15.1/SE; Stata Corporation, College Station, Texas, USA).

\section{Results}

\section{Study Population}

The selection of the 115 patients with acquired HIV-1 drug resistance (ADR) after cART initiation is depicted in the flow chart [Supplementary Figure 1]. All patients started directly with $\geq 3$ drugs from at least two different drug classes. The year of cART initiation was subdivided in 3 categories: (1) before 1999 for 6 (1.7\%), (2) between 1999-2006 for 78 (67.8\%) and (3) after 2006 for 31 patients (27\%). 
Overall 91 (79.1\%) cases and 93 (80.9\%) controls had a baseline viral load > 10’000 copies/ml. The median [IQR] baseline viral load was 5.2 [4.9 - 5.7] and 5.1 [4.7-5.6] $\log _{10}$ RNA copies/mL in cases and controls, respectively. High viral loads above $100^{\prime} 000$ copies $/ \mathrm{mL}$ were found in $65 \%$ of controls and $57 \%$ of cases. Pre-treatment drug resistance mutations were found in a minority of patients $(\mathrm{n}=18,7.8 \%)$. In the study population 22 (19\%) cases vs. 38 (33\%) controls were MSM, 38 (33\%) cases vs. 45 (39\%) controls were heterosexual men, 43 (37\%) cases vs. 27 (23\%) controls were heterosexual women. In the transmission group IDU 12 (10.4\%) cases vs. 5 (4.3\%) controls were identified.

\section{Sociodemographic factors largely determine the development of ADR}

We identified several risk factors, which strongly correlated with ADR in the univariable as well as in the multivariable model (Figure 1, Supplementary Table 3). First, we focused on origin of the patients and analyzed whether geographical and sociocultural background influenced adherence and ADR. We found a strong correlation with increased risk for developing ADR and African origin (multivariable odds ratio (mOR): 3.4, 95\% CI 1.0-9.2, p 0.047). We further analyzed characteristics of this group (Figure 2 A). We found in the univariable model that being a migrant seeking for asylum was highly associated with ADR (uOR: 2.6, 95\% CI: 1.1-6.0, p<0.023), as well as having a language barrier (uOR: 2.6, 95\% CI: 1.4-4.7, $\mathrm{p}=0.002$ ) or being female (uOR: $2.2,95 \% \mathrm{CI}: 1.2-3.8, \mathrm{p}=0.007$ ). Because of collinearity with the origin, we did not include these factors in the multivariable model.

We also analyzed socioeconomic factors and found that unemployment bears a relevant risk (mOR 2.9 95\% CI: 1.3-6.4, p 0.008) (Figure 1, Supplementary Table 3). In a more detailed analysis of this subgroup (Figure 2 B), limited education (uOR: 3.3, 95\% CI: 1.4-7.7, p=0.006), being an IDU (uOR: 3.9, 95\% CI: 1.2-12.9, p<0.021) and excessive consumption of alcohol were associated with ADR (uOR: 3.0, 1.3-7.2, $\mathrm{p}<0.013)$. Because of the collinearity of these factors with unemployment, we did not include them in the multivariable model.

On the contrary, having a stable income and being able to work $>50 \%$ of the time was a strongly protective factor (uOR: 0.27, 95\% CI: 0.14-0.52, p<0.001). 
Overall, older age tended to have a protective effect upon ADR development (mOR per 10 years increase: 0.73, 95\% CI 0.5-1.0, p=0.073) (Figure 1, Supplementary Table 3).

Importantly, when we analyzed only patients with a perfect match ( $\mathrm{N}=144)$, we had an almost identical result, which confirms the robustness of the analysis (data not shown).

\section{Mental health issues severely impacts ADR}

We found that mental health issues correlated with ADR (Figure 1, Supplementary Table 3). A clear association of ADR to the documented diagnosis of depression (uOR 2.0, 95\% CI 1.1, 3.8, p=0.029) (Table 1) was ascertained. We analyzed whether side effects had an influence upon adherence and subsequently on ADR. We therefore additionally assessed side effects which can associate with mental health disorders and which are not included in the SHCS questionnaire. Nightmares (uOR 4.0,95\% CI 1.41, 7.66, p=0.007), mood swings (uOR 4.1, 95\% CI 1.8, 9.4, p=0.001) and tiredness (uOR 4.7, 95\% CI 1.6, 13.7, p=0.005) were associated with ADR (Table 1). When analyzing only the subgroup of patient with diagnosed mental health disorders and which are in psychiatric treatment in the multivariable model, the key findings were corroborated (Supplementary Table 5).

\section{Co-medication with anti-infectives increases ADR}

We also studied whether a higher pill burden with co-medication led to an increased risk of ADR. We found that co-medication with anti-infectives (e.g. antibiotic, antimycobacterial, antifungal, antiviral (other than ART), antiparasitic medication) correlated with ADR (mOR 3.6 (95\%CI: 1.0-12.6), p=0.045).

We were not able to assess whether specific anti- infective therapy were leading to ADR as the sample size of the different subgroups was too small. We also analyzed whether there were possible drug-drug interactions or drug level measurements that explained why ADR developed in patients with antiinfectives. However, no obvious drug-drug interactions were noticed and drug levels were rarely measured (only in 47 patients). 


\section{Adherence is associated with ADR}

As expected, low self- reported adherence is closely associated with the occurrence of drug resistance. Compared to controls, more cases missed the dose at least once a month (18.3\% vs $15.7 \%)$ or at least once a week (36.5\%vs. 2.6\%). We did a sensitivity analysis including adherence in the multivariable model. The risk was 3.7 (95\% CI: 1.2-11.6) and 9.4 (95\% CI: 2.4-36.5) times increased among patients who missed the dose at least once a month or at least once a week, compared to patients who never missed a dose (Supplementary Table 4). However, we cannot confirm that it is associated with barriers to care as shown in other studies $[14,19,32]$. On the contrary, in our cohort we detected higher number of visits (> 4 in 12 months) being correlated to ADR (OR 2.67, 95\% CI 1.37, 5.18, p 0.004). This shows that in the SHCS patients at risk are more closely followed up before detection of ADR. However, the increased visit frequency seem not to be sufficient to avoid ADR. Whether low SES favored also an avoidance coping was not explored in this study.

\section{Unexplained cases of ADR}

Remarkably, patients with ADR and without any risk factor identified in the multivariable model (African origin, unemployment, co-medication with anti-infectives, mental health issues, age $<45$ years) were rarely identified ( $n=5$ (4\%). Patients with ADR had a median (IQR) of $3(2-4)$ of the mentioned risk factors in the multivariable model (Figure 1).

\section{Discussion}

Although ADR has become very rare in Switzerland since the introduction of cART [6], full access to antiretroviral treatment, viral load monitoring as well as resistance testing, there are still individuals that experience treatment failure due to ADR. In this study, we clearly identified risk factors for emergence of drug resistance in this generally highly successfully treated patient population.

One of the key results our study reveals is that the patient's origin and sociocultural background largely affect treatment outcome. Being of black ethnicity, originating from Africa and arriving to Switzerland as 
migrant seeking for asylum affects treatment success and leads to more ADR. We also show that this is further aggravated when difficulties in communication with the health care team due to language barrier are met.

When analyzing the influence of gender in the univariable model, being a woman is clearly more often associated with ADR. A possible explanation for this result may be the myriad of psychosocial stressors observed in women living with HIV, such as caregiving and household responsibilities, the already described financial related stressors and relationship problems [33-36]. In summary, additional to the psychosocial stressors of - mostly female- migrants, impaired communication and therefore obstacles due to misunderstanding largely affect treatment success.

We also show that unemployment and limited education is associated with higher likelihood of developing drug resistance. Our findings support previous findings that low SES is associated with lower drug adherence [15-17]. However, we cannot confirm that it is associated with barriers to care as shown in other studies [14,32]. On the contrary, the increased visit numbers before ADR show, that a problem in adherence and incumbent risk were noticed and a strong effort was put to improve patient care. Nevertheless, it shows that the mechanisms in place still were not strong enough to prevent ADR. These findings also highlight that different subgroups can experience similar stressors, which negatively impact adherence and subsequently lead to ADR.

Psychological symptoms have been recognized to interfere with activities of daily life, physical functioning, interpersonal relationships and adherence [36, 37]. Our study shows a significant correlation between mental health issues and ADR. Also, the presence of side-effects associated with mental health issues or even disguising psychiatric problems (e.g. tiredness , mood swings, nightmares) also correlated with ADR. The described symptoms could therefore reflect an underlying and undiagnosed mental disorder- rather than ART associated side effects. Remarkably, no association was found to psychiatric treatment or co-medication with antidepressant. With the clear association of mental health issues and ADR, future improvement of patient care needs to focus not only on diagnosis mental health disorders but 
also adequate treatment. Overall, our results corroborate previous findings showing a clear association between depression and non-adherence to ART $[28,38]$.

We found an association with co-medication with anti-infectives. Polypharmacy impacts how drugs are tolerated, increases possible drug interaction and most importantly also leads to reduced adherence [39, 40]. The association of anti-infectives with ADR might hint to more complicated drug regimens, more side effects and indirectly also towards more drug-drug interactions explaining this finding. In these patients with more complicated course of HIV infections, a more regular and close guidance during the time of multiple therapies is advised. [41].

The study has been systematically conducted in a prospectively followed, well characterized, representative patient population in a real world setting with full access, monitoring and resistance testing for all (at least 75\% of all ART treated adults in Switzerland are enrolled in the SHCS[6]), which allowed a careful matched case control study and a detailed chart review. However, the study has limitations. The retrospective analysis of patient charts and the varying quality of documentation and sometimes missing information may have influenced the results. Moreover, the diagnosis of mental health issues was based on documentation by the treating infectious disease specialist and could have been incompletely documented. Importantly, the documentation and diagnosis of mental health issues will improve, as the SHCS cohort has implemented an exact questionnaire addressing these topics since 2014.

In conclusion, we show that ADR is still of concern in the era of cART in vulnerable patient groups that face a multitude of challenges. The factors we identified here, i.e. lower education status, psychiatric comorbidities, being a migrant seeking for asylum, will help practitioners identify these patients early on. However, multidisciplinary efforts are likely needed to help patients to overcome these significant barriers to adherence, and might include social services, counseling, or a referral to a psychiatrist. This study suggests that with improved patient care and previous knowledge of the identified risk factors a further reduction of ADR can be achieved. 


\section{Author's contributions}

Conceived and designed the study: IA, AUS,, HFG, Performed the analysis: IA and AUS, Collected and contributed data: IA, AUS, JB, SY, TK, MP, MB, JF, AC, PS, MC, EB, HFG, Wrote the paper: IA, AS, HFG. All authors read and approved the final manuscript.

\section{Acknowledgements}

We thank the patients who participate in the Swiss HIV Cohort Study; the physicians and study nurses, for excellent patient care; the resistance laboratories, for high-quality genotyping drug resistance testing; SmartGene (Zug, Switzerland), for technical support; Alexandra Scherrer, Susanne Wild, Anna Traytel from the SHCS data center for data management, Danièle Perraudin and Mirjam Minichiello for administration. The members of the Swiss HIV Cohort Study include the following: Anagnostopoulos A, Battegay M, Bernasconi E, Böni J, Braun DL, Bucher HC, Calmy A, Cavassini M, Ciuffi A, Dollenmaier G, Egger M, Elzi L, Fehr J, Fellay J, Furrer H (Chairman of the Clinical and Laboratory Committee), Fux CA, Günthard HF (President of the SHCS), Haerry D (deputy of "Positive Council"), Hasse

B, Hirsch HH, Hoffmann M, Hösli I, Huber M, Kahlert C, Kaiser L, Keiser O, Klimkait T, Kouyos RD, Kovari H, Ledergerber B, Martinetti G, Martinez de Tejada B, Marzolini C, Metzner KJ, Müller N, Nicca D, Paioni P, Pantaleo G, Perreau M, Rauch A (Chairman of the Scientific Board), Rudin C (Chairman of the Mother \& Child Substudy), Scherrer AU (Head of Data Centre), Schmid P, Speck R, Stöckle M, Tarr P, Trkola A, Vernazza P, Wandeler G, Weber R, Yerly S.

\section{Funding}

This work was supported by the Swiss National Science Foundation (Grant \# 179571 to HFG) and was financed within the framework of the Swiss HIV Cohort Study, supported by the Swiss National Science Foundation (grant \#148522), by the SHCS research foundation by the Yvonne Jacob Foundation (to HFG), by the clinical research priority program of the University of Zurich "Viral infectious diseases, ZPHI" (to HFG). HFG has received an unrestricted research Grant from Gilead to the SHCS Research Foundation.

\section{Conflicts of interests}


HFG has received unrestricted research grants from Gilead Sciences and Roche; fees for data and safety monitoring board membership from Merck; consulting/advisory board membership fees from Gilead Sciences, Sandoz and Mepha, and grants from Systems.X, and NIH. MB has received research or educational grants by Abbvie AG, Gilead Sciences Switzerland Sàrl, Janssen-Cilag AG, MSD Merck Sharp \& Dohme AG and ViiV Healthcare GmbH. HF reports support to his institution for advisory boards and/or travel grants and/or educational grants from Janssen-Cilag, MSD, Gilead Sciences, Abbvie, and BristolMyers Squibb, Viiv, Sandoz, and an unrestricted research grant from Gilead Sciences. AC has received unrestricted educational and research grants from MSD, Gilead and ViiVAll remuneration went to his home institution and not to AC personally, and all remuneration was provided outside the submitted work. MC has received research and travel grants for his institution from ViiV and Gilead, as well as payments from Abbvie and MSD. EB has received fees for his institution for participation to advisory board from MSD, Gilead Sciences, ViiV Healthcare, Sandoz, Pfizer, Abbvie and Janssen. 


\section{References}

1. Gulick, R.M., et al., Treatment with indinavir, zidovudine, and lamivudine in adults with human immunodeficiency virus infection and prior antiretroviral therapy. $\mathrm{N}$ Engl J Med, 1997. 337(11): p. 734-9.

2. von Wyl, V., et al., Emergence of HIV-1 drug resistance in previously untreated patients initiating combination antiretroviral treatment: a comparison of different regimen types. Arch Intern Med, 2007. 167(16): p. 1782-90.

3. Dunn, D., et al., Population trends in the prevalence and patterns of protease resistance related to exposure to unboosted and boosted protease inhibitors. Antivir Ther, 2008. 13(6): p. 771-7.

4. Bontell, I., et al., Trends in antiretroviral therapy and prevalence of HIV drug resistance mutations in Sweden 1997-2011. PLoS One, 2013. 8(3): p. e59337.

5. Buchacz, K., et al., Trends in Decline of Antiretroviral Resistance among ARV-Experienced Patients in the HIV Outpatient Study: 1999-2008. AIDS Res Treat, 2012. 2012: p. 230290.

6. Scherrer, A.U., et al., Emergence of Acquired HIV-1 Drug Resistance Almost Stopped in Switzerland: A 15-Year Prospective Cohort Analysis. Clin Infect Dis, 2016. 62(10): p. 13101317.

7. Gunthard, H.F., et al., Human Immunodeficiency Virus Drug Resistance: 2018 Recommendations of the International Antiviral Society-USA Panel. Clin Infect Dis, 2018.

8. Cameron, D.W., et al., Randomised placebo-controlled trial of ritonavir in advanced HIV-1 disease. The Advanced HIV Disease Ritonavir Study Group. Lancet, 1998. 351(9102): p. 543-9.

9. Hammer, S.M., et al., A controlled trial of two nucleoside analogues plus indinavir in persons with human immunodeficiency virus infection and CD4 cell counts of 200 per cubic millimeter or less. AIDS Clinical Trials Group 320 Study Team. N Engl J Med, 1997. 337(11): p. 725-33.

10. Gunthard, H.F., et al., Antiretroviral treatment of adult HIV infection: 2014 recommendations of the International Antiviral Society-USA Panel. JAMA, 2014. 312(4): p. 410-25.

11. Antiretroviral Therapy Cohort, C., Life expectancy of individuals on combination antiretroviral therapy in high-income countries: a collaborative analysis of 14 cohort studies. Lancet, 2008. 372(9635): p. 293-9.

12. Gueler, A., et al., Life expectancy in HIV-positive persons in Switzerland: matched comparison with general population. AIDS, 2017. 31(3): p. 427-436. 
13. Katz, I.T. and B. Maughan-Brown, Improved life expectancy of people living with HIV: who is left behind? Lancet HIV, 2017. 4(8): p. e324-e326.

14. Rocheleau, G., et al., Sociodemographic correlates of HIV drug resistance and access to drug resistance testing in British Columbia, Canada. PLoS One, 2017. 12(9): p. e0184848.

15. Kinsler, J.J., et al., The effect of perceived stigma from a health care provider on access to care among a low-income HIV-positive population. AIDS Patient Care STDS, 2007. 21(8): p. 584-92.

16. Cunningham, C.O., et al., Utilization of health care services in hard-to-reach marginalized HIV-infected individuals. AIDS Patient Care STDS, 2007. 21(3): p. 177-86.

17. Leaver, C.A., et al., The effects of housing status on health-related outcomes in people living with HIV: a systematic review of the literature. AIDS Behav, 2007. 11(6 Suppl): p. 85-100.

18. Chambers, L.A., et al., Stigma, HIV and health: a qualitative synthesis. BMC Public Health, 2015. 15: p. 848.

19. Giordano, T.P., et al., Retention in care: a challenge to survival with HIV infection. Clin Infect Dis, 2007. 44(11): p. 1493-9.

20. Burch, L.S., et al., Socioeconomic status and response to antiretroviral therapy in highincome countries: a literature review. AIDS, 2016. 30(8): p. 1147-62.

21. Larder, B.A., G. Darby, and D.D. Richman, HIV with reduced sensitivity to zidovudine (AZT) isolated during prolonged therapy. Science, 1989. 243(4899): p. 1731-4.

22. Wensing, A.M., et al., 2017 Update of the Drug Resistance Mutations in HIV-1. Top Antivir Med, 2017. 24(4): p. 132-133.

23. Murphy, R.A., et al., Outcomes after virologic failure of first-line ART in South Africa. AIDS, 2010. 24(7): p. 1007-12.

24. Swiss, H.I.V.C.S., et al., Cohort profile: the Swiss HIV Cohort study. Int J Epidemiol, 2010. 39(5): p. 1179-89.

25. Yang, W.L., et al., Assessing the Paradox Between Transmitted and Acquired HIV Type 1 Drug Resistance Mutations in the Swiss HIV Cohort Study From 1998 to 2012. J Infect Dis, 2015. 212(1): p. 28-38.

26. Anagnostopoulos, A., et al., Frequency of and Risk Factors for Depression among Participants in the Swiss HIV Cohort Study (SHCS). PLoS One, 2015. 10(10): p. e0140943.

27. Bing, E.G., et al., Psychiatric disorders and drug use among human immunodeficiency virusinfected adults in the United States. Arch Gen Psychiatry, 2001. 58(8): p. 721-8.

28. Gonzalez, J.S., et al., Depression and HIV/AIDS treatment nonadherence: a review and meta-analysis. J Acquir Immune Defic Syndr, 2011. 58(2): p. 181-7. 
29. Glass, T.R., et al., Determinants of sustained viral suppression in HIV-infected patients with self-reported poor adherence to antiretroviral therapy. PLoS One, 2012. 7(1): p. e29186.

30. von Wyl, V., et al., Adherence as a predictor of the development of class-specific resistance mutations: the Swiss HIV Cohort Study. PLoS One, 2013. 8(10): p. e77691.

31. Glass, T.R., et al., Self-reported nonadherence to antiretroviral therapy as a predictor of viral failure and mortality. AIDS, 2015. 29(16): p. 2195-200.

32. Giordano, T.P., Retention in HIV care: what the clinician needs to know. Top Antivir Med, 2011. 19(1): p. 12-6.

33. Durvasula, R., HIV/AIDS in older women: unique challenges, unmet needs. Behav Med, 2014. 40(3): p. 85-98.

34. Catz, S.L., C. Gore-Felton, and J.B. McClure, Psychological distress among minority and lowincome women living with HIV. Behav Med, 2002. 28(2): p. 53-60.

35. Cederfjall, C., et al., Gender differences in perceived health-related quality of life among patients with HIV infection. AIDS Patient Care STDS, 2001. 15(1): p. 31-9.

36. Ickovics, J.R., et al., Mortality, CD4 cell count decline, and depressive symptoms among HIVseropositive women: longitudinal analysis from the HIV Epidemiology Research Study. JAMA, 2001. 285(11): p. 1466-74.

37. Arseniou, S., A. Arvaniti, and M. Samakouri, HIV infection and depression. Psychiatry Clin Neurosci, 2014. 68(2): p. 96-109.

38. Starace, F., et al., Depression is a risk factor for suboptimal adherence to highly active antiretroviral therapy. J Acquir Immune Defic Syndr, 2002. 31 Suppl 3: p. S136-9.

39. Kamal, S., et al., HIV-infected patients' beliefs about their chronic co-treatments in comparison with their combined antiretroviral therapy. HIV Med, 2018. 19(1): p. 49-58.

40. Marzolini, C., et al., Ageing with HIV: medication use and risk for potential drug-drug interactions. J Antimicrob Chemother, 2011. 66(9): p. 2107-11.

41. Ballester-Arnal, R., et al., A Spanish Study on Psychological Predictors of Quality of Life in People with HIV. AIDS Behav, 2016. 20(2): p. 281-91. 
Table 1: Univariable conditional logistic regression looking at the association of mental health issues and acquired drug resistance

\begin{tabular}{|c|c|c|c|c|c|}
\hline & $\begin{array}{l}\text { Control } \\
(n=115)\end{array}$ & $\begin{array}{l}\text { Cases } \\
(n=115)\end{array}$ & $\mathrm{uOR}$ & $(95 \% \mathrm{CI})$ & p-value \\
\hline \multicolumn{6}{|c|}{ Depression } \\
\hline No & $90(78 \%)$ & $74(64 \%)$ & Ref & & \\
\hline Yes & $23(20 \%)$ & $37(32 \%)$ & 2.0 & $(1.1-3.8)$ & 0.029 \\
\hline Unknown & $2(2 \%)$ & $4(3 \%)$ & 2.6 & $(0.5-14.6)$ & 0.286 \\
\hline \multicolumn{6}{|c|}{$\begin{array}{l}\text { Psychiatric } \\
\text { treatment }\end{array}$} \\
\hline No & $92(80 \%)$ & $81(70 \%)$ & Ref & & \\
\hline Yes & $21(18 \%)$ & $31(27 \%)$ & 1.6 & $(0.9-3.0)$ & 0.117 \\
\hline Unknown & $2(2 \%)$ & $3(3 \%)$ & 1.8 & $(0.3-11.3)$ & 0.513 \\
\hline \multicolumn{6}{|c|}{ Mood swings } \\
\hline No & $\begin{array}{l}101 \\
(88 \%)\end{array}$ & $66(57 \%)$ & Ref & & \\
\hline Yes & $9(8 \%)$ & $27(23 \%)$ & 4.1 & $(1.8-9.4)$ & 0.001 \\
\hline Unknown & $5(4 \%)$ & $22(19 \%)$ & 6.7 & $(2.2-20.3)$ & 0.001 \\
\hline \multicolumn{6}{|c|}{ Nightmares } \\
\hline No & $\begin{array}{l}102 \\
(89 \%)\end{array}$ & $72(63 \%)$ & Ref & & \\
\hline Yes & $8(7 \%)$ & $21(18 \%)$ & 4.0 & $(1.4-11.0)$ & 0.007 \\
\hline Unknown & $5(4 \%)$ & $22(19 \%)$ & 5.7 & $(1.9-16.8)$ & 0.002 \\
\hline \multicolumn{6}{|c|}{ Sleeping disorder } \\
\hline No & $95(83 \%)$ & $69(60 \%)$ & Ref & & \\
\hline Yes & $15(13 \%)$ & $24(21 \%)$ & 2.2 & $(1.0-4.7)$ & 0.056 \\
\hline Unknown & $5(4 \%)$ & $22(19 \%)$ & 5.7 & $(1.9-16.7)$ & 0.002 \\
\hline
\end{tabular}




\begin{tabular}{|l|l|l|l|l|l|}
\hline Tiredness & & & & & \\
\hline No & $\begin{array}{l}102 \\
(89 \%)\end{array}$ & $73(63 \%)$ & Ref & & \\
\hline Yes & $5(4 \%)$ & $16(14 \%)$ & 4.7 & $(1.6-13.7)$ & 0.005 \\
\hline Unknown & $8(7 \%)$ & $26(23 \%)$ & 4.6 & $(1.9-11.3)$ & 0.001 \\
\hline Alcohol abuse & $\begin{array}{l}100 \\
(87 \%)\end{array}$ & $82(71 \%)$ & Ref & & \\
\hline No & $7(6 \%)$ & $20(17 \%)$ & 3.0 & $(1.3-7.2)$ & 0.013 \\
\hline Yes & $8(7 \%)$ & $13(11 \%)$ & 1.9 & $(0.7-5.0)$ & 0.172 \\
\hline Unknown & & & & & \\
\hline
\end{tabular}




\section{Figure legends}

Figure 1: Risk factors for acquired drug resistance under ART. Univariable and multivariable conditional logistic regression

Figure 2: Detailed analyses of sociocultural and socioeconomic factors based on origin (A) and job situation (B). p-values are based on Fisher's exact test 
Figure 1

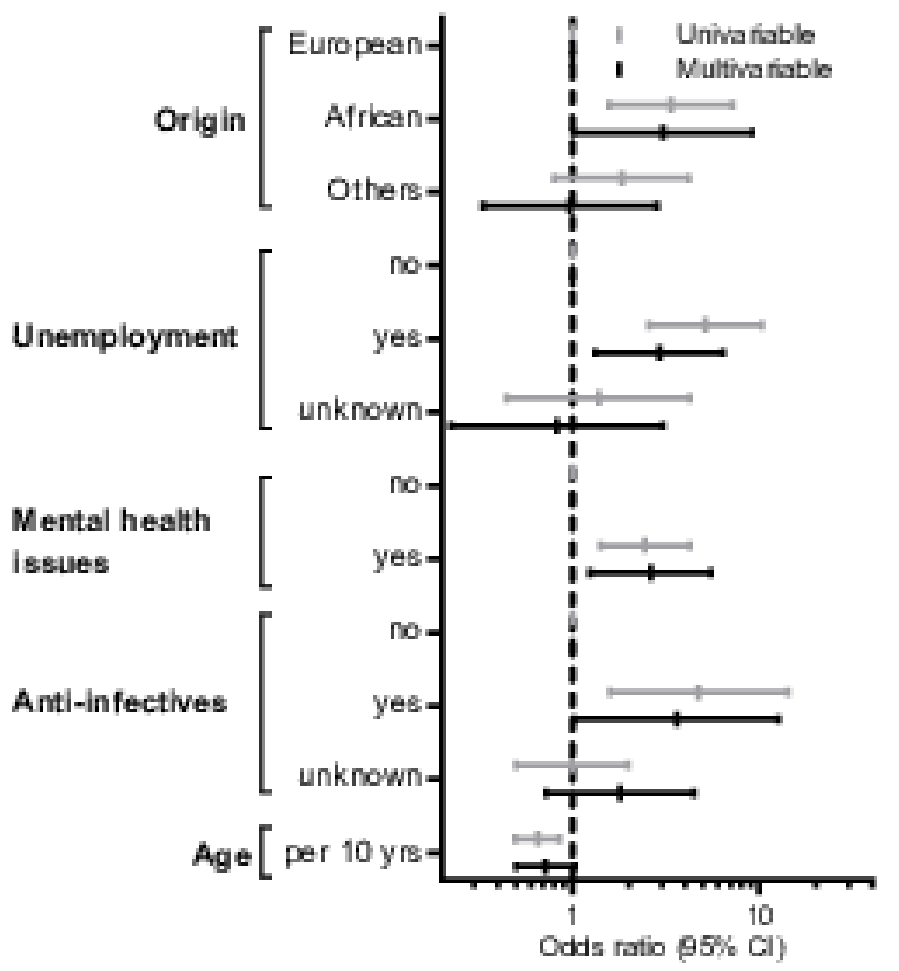


Figure 2

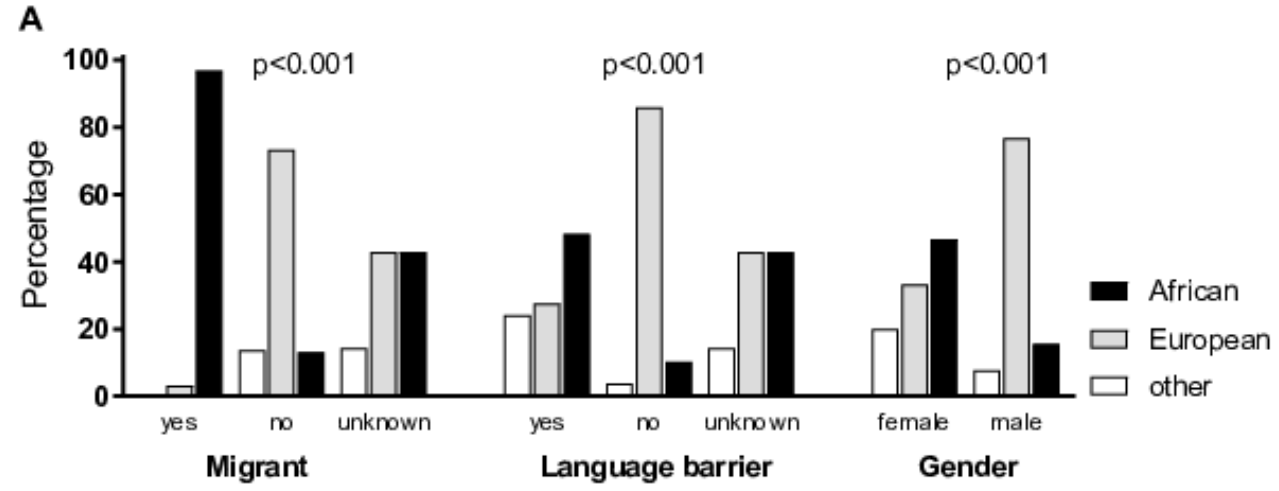

B

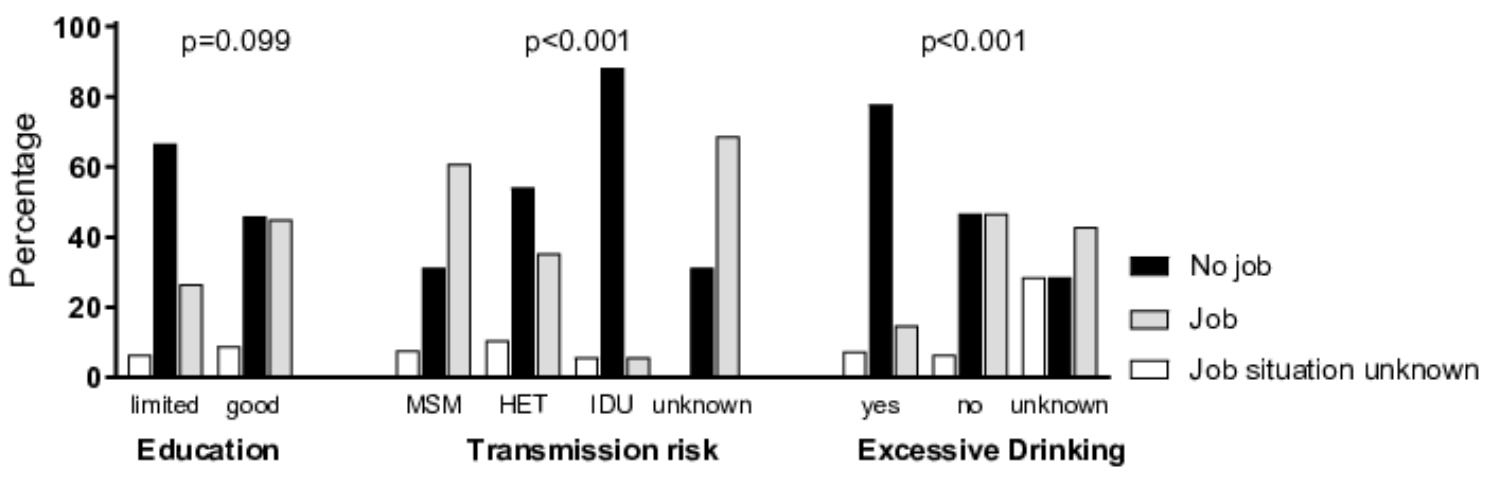

